\title{
Burden of Migraine in Patients With Preventive Treatment Failure Attending European Headache Specialist Centers: Real-World Evidence From the BECOME Study
}

\author{
Patricia Pozo-Rosich • Christian Lucas - David P. B. Watson • \\ Charly Gaul · Emma Ramsden · Shannon Ritter • Paolo Martelletti • \\ Josefin Snellman
}

Received: July 28, 2021 / Accepted: September 24, 2021 / Published online: October 13, 2021

(C) The Author(s) 2021

\section{ABSTRACT}

Introduction: Migraine is consistently ranked as one of the most disabling neurological conditions in the world, often causing a substantial impairment of daily activities and quality of life. It also carries a high economic burden of

Supplementary Information The online version contains supplementary material available at https://doi. org/10.1007/s40122-021-00331-3.

\section{P. Pozo-Rosich $(\bowtie)$}

Headache Unit, Neurology Department, Vall d'Hebron University Hospital, Pg. Vall d'Hebron, 119-129, 08035 Barcelona, Spain

e-mail:ppozo@vhebron.net

P. Pozo-Rosich

Headache and Neurological Pain Research Group, Vall d'Hebron Institute of Research (VHIR),

Universitat Autònoma de Barcelona, 08035

Barcelona, Spain

C. Lucas

Pain Clinic, Service de Neurochirurgie, Hôpital Salengro, CHU de Lille, 59037 Lille Cedex, France e-mail: christian.lucas@chru-lille.fr

D. P. B. Watson

Hamilton Medical Group, Aberdeen AB15 4ZT, Scotland

e-mail: david.watson@nhs.net

C. Gaul

Migraine and Headache Clinic Königstein, 61462

Königstein im Taunus, Germany

e-mail: c.gaul@kopfschmerz-frankfutr.de direct and indirect healthcare costs. Patients with difficult-to-treat migraine often cycle through different preventive therapies, but realworld prospective evidence describing the burden of migraine in patients with prior preventive treatment failure (PPTF) in Europe is limited. In BECOME, we aimed to characterize and assess the prevalence and burden of migraine in patients with PPTF attending specialist headache centers in Europe and Israel. Furthermore, we assessed this burden in pre-

E. Ramsden · J. Snellman

Novartis Pharma AG, 4033 Basel, Switzerland

E. Ramsden

e-mail: CTEConsult@outlook.com

\section{J. Snellman}

e-mail: josefin.snellman@novartis.com

\section{S. Ritter}

Novartis Pharmaceuticals Corporation, East

Hanover, NJ 07936, USA

e-mail: shannon.ritter@novartis.com

\section{P. Martelletti}

Department of Clinical and Molecular Medicine, Sant'Andrea Hospital, Sapienza University of Rome, 00185 Rome, Italy

\section{P. Martelletti}

Regional Referral Headache Center, Sant'Andrea

Hospital, 00189 Rome, Italy

e-mail: paolo.martelletti@uniroma1.it 
specified subgroups based on the frequency of monthly migraine days (MMD) and number of PPTFs.

Methods: BECOME was a prospective, non-interventional study conducted in two concurrent parts across 17 countries in Europe and Israel. In part 1, patients visiting the centers over a 3-month period were screened for frequency of PPTF, MMD, and other characteristics. In part 2, patients from part 1 with $\geq 1$ PPTF and $\geq 4 \mathrm{MMD}$ were enrolled, and impact of migraine on patient-reported outcomes, and healthcare resource utilization (HRU) were examined.

Results: In part $1 \quad(n=20,837), 62.2 \%$ of patients reported $\geq 1$ PPTF. In part $2(n=2419)$, $15.3 \%$ of patients reported $\geq 4$ PPTF. In part 2 , the migraine burden measured by the EuroQoL 5 dimensions 5 level (EQ-5D-5L) questionnaire indicated an impact of at least moderate severity in performing usual activities in $26.5 \%$ of patients, pain/discomfort in $51.2 \%$, and $26.1 \%$ reported being at least moderately anxious/depressed. Most patients reported a severe impact on daily activities and disability due to migraine. Abnormal Hospital Anxiety and Depression subscale scores of $\geq 11$ were observed in 29\% (anxiety) and 19.8\% (depression) of the population. In part 2, analysis of HRU showed $21.2 \%$ patients visited an emergency department and $8.4 \%$ were hospitalized for headache/migraine in the past year.

Conclusions: This study provides real-world evidence of the high personal, social, and HRU burden of migraine in Europe and Israel.

Keywords: Burden; Healthcare resource; Migraine; Patient-reported outcomes; Treatment failure; Work productivity

\section{Key Summary Points}

Migraine is consistently ranked as one of the most disabling conditions in the world, associated with functional impairment affecting family, work, social life and with a substantial personal, social, and healthcare economic burden.
Patients who have previously failed migraine preventive therapies represent a difficult-to-treat population, in whom these burdens may be even greater.

In BECOME, we aimed to determine the prevalence of patients with migraine and at least one prior preventive treatment failure (PPTF) attending specialist headache centers in Europe and Israel, understand their disease characteristics, assess the burden of disease on quality of life (QoL) and healthcare resource utilization (HRU), and explore whether the burden is associated with frequency of monthly migraine days (MMD) and/or PPTF.

The study identified a high prevalence of PPTF among patients with migraine who visited specialist headache clinics and demonstrated that patients suffered severe impact on their QoL and on their personal, professional, and social activities as measured by patient-reported outcomes (PRO).

Overall, $22 \%$ of participants in BECOME had a psychiatric comorbidity, with depression and anxiety accounting for $17 \%$ of this comorbidity, suggesting an intrinsic link between migraine and psychiatric comorbidity; furthermore both the number of MMD and PPTFs were associated with the prevalence of psychiatric comorbidities.

The prevalence of hospitalizations associated with migraine in the previous 12 months increased with increases in both MMD and PPTF and strikingly, reached $>17 \%$ among those classed as having chronic migraine and with $\geq 4$ PPTFs.

These findings indicate a significant interplay between MMD frequency and PPTFs in increasing the burden of migraine and indicate an unmet need for more effective preventive therapy for this difficult-to-treat population. 


\section{DIGITAL FEATURES}

This article is published with digital features, including a video abstract, to facilitate understanding of the article. To view digital features for this article go to https://doi.org/10.6084/ m9.figshare.16676617.

\section{INTRODUCTION}

Migraine, a debilitating neurological disease, is the second leading cause of years lived with disability (YLD) worldwide and the leading cause of YLD in people aged $<50$ years in Europe $[1,2]$. It is often difficult-to-treat with a significant proportion of patients cycling through different preventive medications. While the burden of migraine in Europe has been previously reported [3-6], the evidence relating to the burden in patients with prior preventive treatment failure (PPTF) is limited. Furthermore, an acknowledged limitation of these burden studies has been the extent to which migraine has been correctly diagnosed in all cases. Therefore, the current study focuses on patients with PPTF attending headache specialist centers, in which the chances of misdiagnosis are reduced. In this study, we aimed to describe the disease characteristics, especially in terms of monthly migraine days (MMD) and treatment failures, among patients who visited specialty care centers across Europe and Israel. Additionally, we investigated the disease burden and healthcare resource utilization (HRU) among patients who had failed standard of care preventive treatments.

\section{METHODS}

\section{Study Design}

BECOME (Burden of migrainE in specialist headache Centers treating patients with prOphylactic treatMent failurE) was a prospective, multicenter, non-interventional, cross-sectional study conducted in two concurrent parts between 27 November 2017 and 5 October 2018 (before the launch of calcitonin gene-related peptide inhibitors) (Fig. 1). BECOME included study sites across Europe and Israel that were considered to meet local standards for headache specialist centers, defined as clinical practices

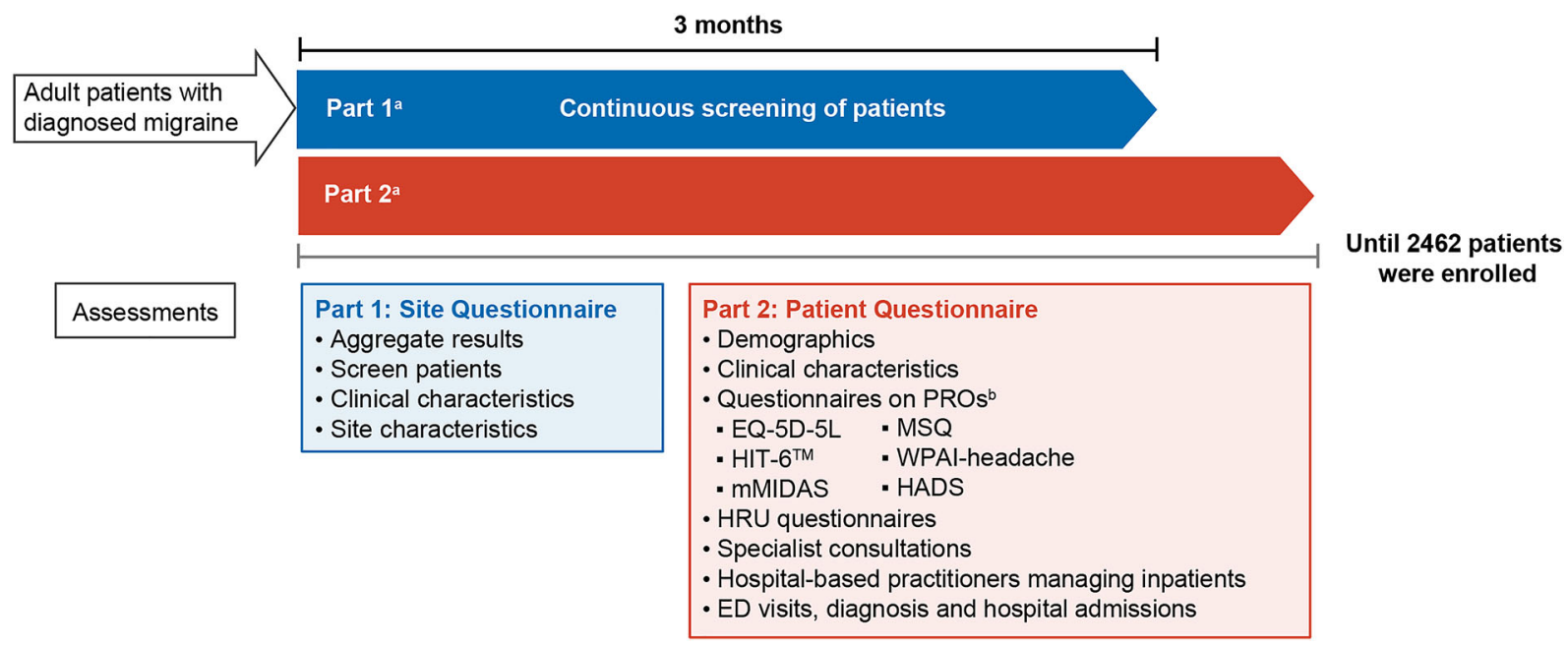

Fig. 1 Design of the BECOME study. ${ }^{\text {a Part }} 1$ and part 2 of the study could be completed on the same day; ${ }^{\mathrm{b}}$ Intended for all patients and completed within 1 day. ED emergency department, EQ-5D-5L EuroQoL 5 dimensions 5 levels, HADS Hospital Anxiety and Depression Scale, HIT-6 headache impact test, HRU healthcare resource utilization, mMIDAS modified migraine disability assessment, MSQ migraine-specific quality of life, PROs patient-reported outcomes, WPAI work productivity and activity impairment 
with at least one physician mainly focused on the treatment of headaches. Site selection was limited to headache specialist centers in order to decrease the risk of incorrect migraine diagnoses and, thereby, an incorrect attribution to migraine of treatment failures and associated burden of disease. Each participating center completed a 20-item site questionnaire, covering the spectrum of disease management, resource availability and skillset, waiting period for new and follow-up patients, and the number of patients referred and treated yearly (for the full list of questions, see Table S1 in the supplementary material).

Part 1 of the study was designed to prospectively assess the prevalence of new and followup patient visits to specialist headache centers and to ascertain the healthcare infrastructure of the participating headache specialist care sites. Each site estimated size and characteristics of their patient population through collection of aggregated data on unique patients with migraine, who were seen as outpatients or inpatients over a 3-month prospective period. Each center also completed a site questionnaire to describe how patients with migraine are managed by the site.

Part 2 of the study was designed to crosssectionally assess the burden of disease and HRU among patients with $\geq 4 \mathrm{MMD}$ and $\geq 1$ PPTF. Eligible patients from part 1 of the study were invited to participate in part 2 , comprising a single visit concurrent with part 1 . Validated questionnaires were used to collect patient-reported measures of the disease burden using both general health and disease-specific tools (Fig. 1). The same structured questionnaires were used for all participating sites and patients across the countries.

\section{Study Participants}

Study participants in part 1 included women and men aged 18-65 years with a diagnosis of migraine. Among them, patients identified in accordance with routine clinical practice were invited to participate in part 2 of the study when they met the criteria of $\geq 4 \mathrm{MMD}$ in the previous 3 months (per the International
Classification of Headache Disorders-3b criteria) [7], a history of at least one previously failed preventive treatment and currently seeking reevaluation due to unsatisfactory treatment in the previous 3 months (the number of patients for whom individual treatments have failed is presented in Table S8 in the supplementary material). PPTF had to have occurred within the past 5 years and was defined as follows: (1) efficacy failure (no meaningful reduction in headache frequency after an adequate period of time at a generally accepted therapeutic dose), (2) tolerability failure with documented discontinuation due to adverse events, and (3) "not suitable for", e.g., due to contraindications or other medically relevant reasons. Patients willing to complete the patient-reported outcome (PRO) questionnaires and provide informed consent were enrolled. Patients participating in any interventional study for migraine were excluded.

\section{Endpoints and Other Variables}

\section{Part 1}

The primary endpoint was the proportion of patients who had failed at least one prior standard of care prophylactic therapy among all patients seeking care at participating European headache specialist care sites within a 3-month period. Other endpoints in part 1 included the proportion of patients visiting headache sites within a 3-month period stratified according to (1) frequency of MMD $(<4,4-7$, and 8-14 MMD, or $\geq 15$ headache days per month, at least eight of which are migraine days), (2) new versus follow-up visit, (3) inpatient versus outpatient, and (4) medication overuse and medication overuse headache [7].

\section{Part 2}

In part 2, PRO questionnaires were administered to assess the disease burden and quality of life (QoL) for pre-specified subgroups based on the frequency of MMD, number of PPTFs, disease duration, and medication overuse. The questionnaires used were EuroQoL 5 dimensions 5 level (EQ-5D-5L), Headache Impact Test6 (HIT-6), modified Migraine Disability 
Assessment (mMIDAS), Migraine-Specific Quality of Life (MSQ), Work Productivity and Activity Impairment for headache (WPAI-headache), and Hospital Anxiety and Depression Scale (HADS). HRU was measured by all-cause or migraine-specific office visits, emergency department (ED) visits, magnetic resonance imaging (MRI) or computed tomography (CT) scans of the head and other investigations, use of migraine medication, evidence of medication overuse, suspected medication overuse headache, and hospitalizations, as assessed by a questionnaire completed by the treating physician during consultation with the patient (for detailed information on PRO scales, see Appendix 1 in the electronic supplementary material).

\section{Subgroup Analysis}

A subgroup analysis was performed to investigate whether the number of PPTFs modulated the burden of migraine associated with the number of MMD corresponding to the classification of episodic migraine (EM) and chronic migraine (CM). PROs and HRU were analyzed in the following EM subgroups: low-frequency EM (LFEM; 4-7 MMD) with 1 PPTF/> 4 PPTFs and high-frequency EM (HFEM; 8-14 MMD) with 1 PPTF/> 4 PPTFs. Furthermore, the burden of migraine was assessed according to $1 \mathrm{PPTF} /$ $>4$ PPTFs in the overall group of patients with $\mathrm{CM}$ and in subgroups designated as low-frequency CM (LFCM; $\geq 15$ monthly headache days [MHD], of which 8-14 are MMD) and highfrequency CM (HFCM; $\geq 15 \mathrm{MHD}$, of which $\geq 15$ are $\mathrm{MMD}$ ). Figure $\mathrm{S} 1$ in the supplementary material summarizes the relationship between these categories. Additionally, the influence of comorbidities on disease burden was assessed.

\section{Statistical Analysis}

A sample size of 2462 patients was determined for part 2 of the study to provide an overall error rate of $1.6 \%$ (half-width of the $95 \%$ confidence interval $[\mathrm{CI}]$ ) when addressing the primary objective (proportion of patients who had failed at least one prior standard of care prophylactic therapy). The Steering Committee estimated the rate of treatment failure to be $>70 \%$ to $100 \%$ depending on the site and therefore a discontinuation rate of $80 \%$ in newly presenting patients at specialist sites for the purposes of sample size calculation. Of particular note is that there was a limit on patient recruitment per country. This limit was estimated to collect data from the minimum number of patients needed to give statistical validity on a per country basis, for the majority of the participating countries. Some countries were too small to achieve this goal, and in those countries a feasibility assessment was done and followed. No statistical hypothesis testing or inferential statistical analyses were performed. Descriptive statistics are used to present the demographics and characteristics of the study population and healthcare centers. Categorical variables are presented as absolute numbers and relative frequencies, and continuous variables as simple statistics. Statistical analyses were conducted using Statistical Analysis System software (SAS version 9.4.3).

\section{Ethics Approval}

The study was approved by an independent ethics committee or relevant institutional review board at all participating centers (Table 99 in the supplementary material) and conducted in accordance with the ethical principles laid down in the Declaration of Helsinki. All participants provided informed consent before study initiation.

\section{RESULTS}

A total of 163 headache specialist centers from 18 countries participated in the study (Fig. 2). A total of 20,837 patients were screened in part 1 of the study and 2488 were enrolled in part 2 , of which 2419 patients were included in the analyses. More than half of these patients $(50.9 \%, 1231 / 2419)$ were recruited from 6 of the 17 countries, namely, Germany, Spain, Italy, Denmark, France, and Greece (Fig. S2 in the supplementary material). For a description of the 163 headache specialist centers 


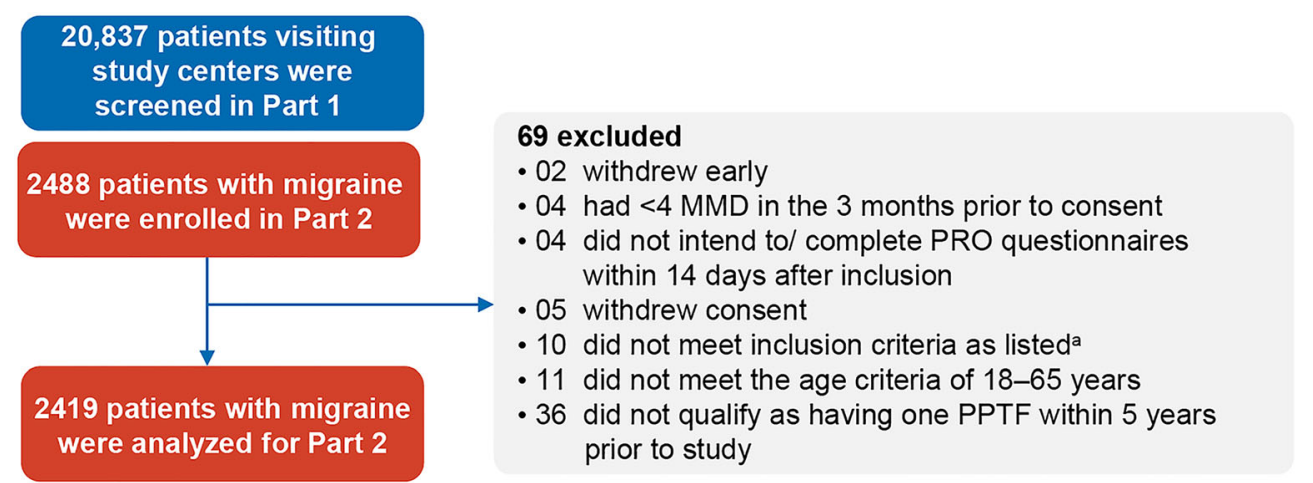

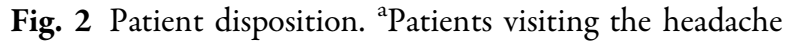
specialist centers as an outpatient (no overnight stay) or an inpatient ( $\geq 1$ overnight stay), as a new patient (first visit), or as a follow-up patient (second or later visit) requiring treatment re-evaluation due to unsatisfactory treatment

participating in the study, refer to Appendix 2 in the electronic supplementary material.

Of the 20,837 patients with migraine examined during the 3 months in part 1 of the study, $74.3 \%(15,479 / 20,837)$ reported more than 4 MMD and $22.9 \%(4767 / 20,837)$ suffered from CM. Medication overuse headache was reported in $11.8 \%(2464 / 20,837)$ of patients. Overall, $62.2 \%(12,957 / 20,837)$ of patients had a history of one or more PPTFs and $16.9 \%(3513 / 20,837)$ of patients had failed at least four preventive treatments. Most patients examined in part 1 visited the centers as outpatients (94.5\% $[19,700 / 20,837])$ and visited for a follow-up (73.0\% [15,216/20,837]) (Table 1).

\section{Study Population in Part 2}

Part 2 of BECOME included 2419 patients with $\geq 4 \mathrm{MMD}$ and $\geq 1 \mathrm{PPTF}$. Among these patients, $41.6 \%(1007 / 2419)$ suffered from CM, whereas $33.3 \%(806 / 2419)$ reported 4-7 MMD and $25.0 \%(605 / 2419)$ had 8-14 MMD. The mean (standard deviation [SD]) age was 43.0 (11.6) years and the majority were women $(86.9 \%$ [2103/2419]) and employed (61.2\% [1480/ 2419]). The average patient had experienced migraine for 23.5 years and $67.8 \%(1641 / 2419)$ of patients had experienced migraine for $>15$ years. The reported mean time from first diagnosis was 15.7 years, suggesting an results in the previous 3 months. MMD monthly migraine days, PPTF prior preventive treatment failure, PRO patient-reported outcome

average gap of 8-10 years between disease onset and diagnosis (Table 1). A history of more than 2 PPTFs was reported by $57.3 \%(1385 / 2419)$ of patients, and $15.3 \%(371 / 2419)$ of patients had failed four or more prophylactic treatments. Among those with $\geq 4$ PPTFs, 55.8\% (207/371) reported CM. The proportion of patients who failed multiple preventive treatments increased with MMD severity (Fig. 3). Among patients with $\geq 4$ PPTFs, 79.8\% (296/371) had > 8 MMD.

\section{Burden of Disease}

Table 2 shows the scores of all PRO questionnaires obtained from patients enrolled in part 2 of the study. General health-related QoL (HRQoL) was reported using the EQ-5D-5L questionnaire. The majority of patients reported no problems in the domains of mobility $(79.7 \%$ [1928/2419]) and self-care (91.2\% [2206/2419]). However, at least moderate problems in performing usual activities were reported in $26.5 \%$ $(642 / 2419)$ of patients, at least moderate pain/ discomfort in $51.2 \%(1239 / 2419)$ of patients, and at least moderate anxiety/depression in $26.1 \%(631 / 2419)$ of patients.

When the EQ-5D-5L responses were analyzed according to the subgroups of disease severity (Table S2 in the supplementary material), the ability to perform usual activities was associated with both MMD and PPTF. Within a given 
Table 1 Characteristics of the BECOME study population

Part $1(N=20,837)$

Characteristics

No PPTF

$\geq 1 \mathrm{PPTF}$

$<4 \mathrm{MMD}$

$\geq 4 \mathrm{MMD}$

Medication overuse

Medication overuse headache

New patient to the center

Follow-up patient to the center

Outpatient visits

Inpatient visits

Unclassified visits

Demographics

Age, mean (SD), years

Women

Married

Employed (full time or part time)

Living independently (alone/with spouse/others)

University education

Clinical characteristics

Migraine duration

$$
<5 \text { years }
$$

6 to 10 years

11 to 15 years

$>15$ years

Time since experiencing the first migraine headache, mean (SD), years

Time since diagnosis of migraine, mean (SD), years

Type of migraine ${ }^{a}$

Migraine without aura

$287(11.9)$

$274(11.3)$

$1641(67.8)$

$23.5(13.2)$

$15.7(11.9)$

Migraine with aura

$1291(53.4)$

Chronic migraine
$290(12.0)$

$731(30.2)$ 
Table 1 continued

Complications of migraine ${ }^{\mathrm{b}}$

Probable migraine

Episodic syndromes that may be associated with migraine

Medication overuse

Medication overuse headache

New patient to the center

Follow-up patient to the center

Inpatient

Outpatient with ED visit

Outpatient without ED visit

Patients with presence of comorbidities

Number of co-morbidities per patient, mean (SD)

Most common comorbidities $(\geq 10 \%)$

Psychiatric disorders

Musculoskeletal and connective tissue disorders

Gastrointestinal disorders

Vascular disorders

Endocrine disorders
$9(0.4)$

$2(0.1)$

$6(0.2)$

$627(25.9)$

$571(23.6)$

$555(22.9)$

$1863(77.0)$

$103(4.3)$

$416(17.2)$

$1899(78.5)$

$1479(61.1)$

$1.3(1.6)$

$532(22.0)$

$322(13.3)$

$250(10.3)$

$249(10.3)$

$246(10.2)$

All values are $n(\%)$, unless indicated

$E D$ emergency department, ICHD International Classification of Headache Disorders, $M M D$ monthly migraine days, $N$ total number of patients in part 1 or part 2, PPTF prior preventive treatment failure, $S D$ standard deviation

${ }^{a}$ Diagnosis according to ICHD-3 code

b Migraine complications that were assessed were status migrainosus, persistent aura without infarction, migrainous infarction, and migraine aura-triggered seizure

MMD category, the proportion of patients who reported at least moderate problems increased with increasing PPTF. A large proportion of patients reported at least moderate pain or discomfort among the subgroups. The disease severity was associated with MMD and also with PPTF. Similarly, for the anxiety or depression domains, the proportion of patients who reported at least moderate anxiety or depression tended to increase with increasing MMD, and no consistent association with PPTF was found.

In the overall part 2 population, $29.0 \%$ (701/ $2419)$ and $19.8 \%(478 / 2419)$ of patients reported abnormal HADS anxiety and depression subscale scores ( $\geq 11$ points), respectively (Table 2). The burden of anxiety and depression increased with MMD frequency and the proportion of patients with HADS anxiety and HADS depression scores in the abnormal range $(\geq 11)$ was greater in the CM subgroups than in the EM subgroups. Interestingly, in both the HFEM and HFCM categories, a higher number of PPTFs was associated with a lower proportion of patients reporting anxiety, perhaps reflecting adaptation to the condition in a population likely to have longer-standing disease. For depression, the proportion of severely affected patients increased with increase in MMD, with 

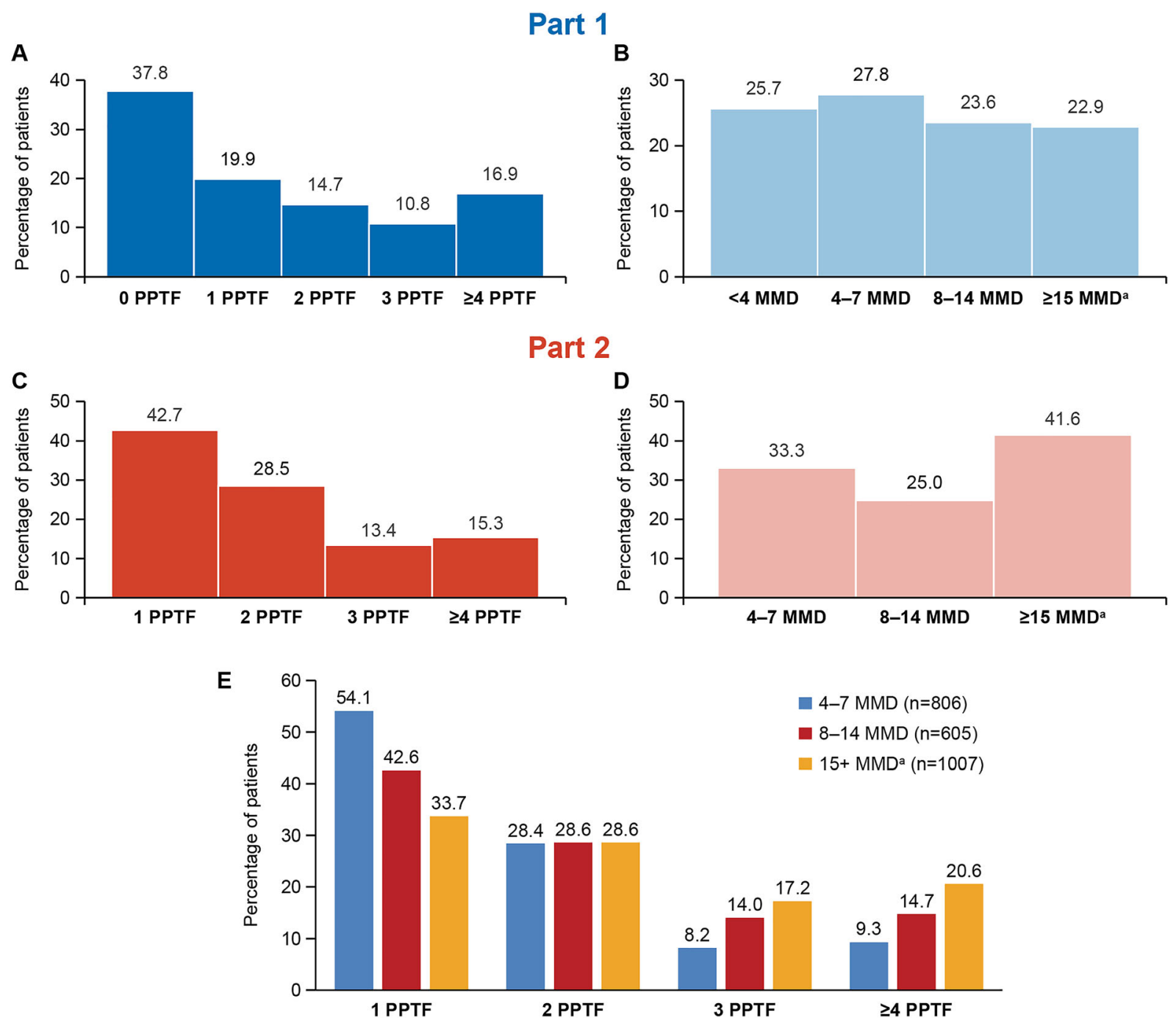

Fig. 3 Frequency of patients with PPTF (a, c), MMD (b, d) in population sets for part $1(N=20,837)$ and part 2 $(N=2419)$, and frequency of patients with MMD by the number of PPTF in part $2(N=2419)(\mathbf{e}) .{ }^{\mathrm{a}} \geq 15$

no effect of treatment failures. Specifically, the proportion of patients with a score of $>8$ on the HADS anxiety and depression subscale followed a similar trend to that observed with the anxiety/depression domain in EQ-5D-5L and seemed to be more closely associated with a higher headache frequency (MMD) than PPTF (Table S2 in the supplementary material).

The severity of the impact of migraine on the daily life of patients was indicated by a mean (95\% CI) HIT-6 score of 65.2 (65.0-65.5) in the overall part 2 population (Table 2 ). In headache days per month with $\geq 8$ MMD. MMD monthly migraine days, $N$ total number of patients in part 1 or part 2; $n$ number of patients; PPTF prior preventive treatment failure

corroboration, the majority of the population $(88 \%, 2129 / 2419)$ indicated severe impact (highest grade on the scale) of migraine on their lives. This finding was in line with the mMIDAS scores in the overall part 2 population, with $81.9 \%(1982 / 2419)$ of patients achieving the severe disability score (grade IV [score $\geq 21]$ ). Findings from subgroup analyses also indicated that the proportion of patients with HIT-6 scores and mMIDAS scores in the more severe categories tended to increase with an increase in $\mathrm{MMD}$, and suggested that within a given MMD 
Table 2 Summary of the total PRO scores and number of patients by domain for the part 2 population $(N=2419)$

\begin{tabular}{|c|c|c|}
\hline Assessment tool & $n$ or $n(\%)$ & Mean $(95 \% \mathrm{CI})$ score \\
\hline EQ-5D-5L utility index score & 2415 & $0.76(0.75$ to 0.77$)$ \\
\hline EQ-5D-5L VAS score & 2419 & $67.3(66.5$ to 68.1$)$ \\
\hline \multicolumn{3}{|l|}{ EQ-5D-5L mobility* } \\
\hline I have no problems in walking about & $1928(79.7)$ & (78.10 to 81.31$)$ \\
\hline I have slight problems in walking about & $311(12.9)$ & $(11.52$ to 14.19$)$ \\
\hline I have moderate problems in walking about & $132(5.5)$ & $(4.55$ to 6.36$)$ \\
\hline I have severe problems in walking about & $40(1.7)$ & $(1.15$ to 2.16$)$ \\
\hline I am unable to walk about & $6(0.2)$ & $(0.05$ to 0.45$)$ \\
\hline Missing & $2(0.1)$ & $(0.00$ to 0.20$)$ \\
\hline \multicolumn{3}{|l|}{ EQ-5D-5L self-care* } \\
\hline I have no problems washing or dressing myself & $2206(91.2)$ & (90.07 to 92.32$)$ \\
\hline I have slight problems washing or dressing myself & $143(5.9)$ & $(4.97$ to 6.85$)$ \\
\hline I have moderate problems washing or dressing myself & $51(2.1)$ & (1.54 to 2.68$)$ \\
\hline I have severe problems washing or dressing myself & $14(0.6)$ & $(0.28$ to 0.88$)$ \\
\hline I am unable to wash or dress myself & $3(0.1)$ & $(0.00$ to 0.26$)$ \\
\hline Missing & $2(0.1)$ & $(0.00$ to 0.20$)$ \\
\hline \multicolumn{3}{|l|}{ EQ-5D-5L usual activities* } \\
\hline I have no problems doing my usual activities & $1229(50.8)$ & $(48.81$ to 52.80$)$ \\
\hline I have slight problems doing my usual activities & $546(22.6)$ & (20.91 to 24.24$)$ \\
\hline I have moderate problems doing my usual activities & $428(17.7)$ & $(16.17$ to 19.21$)$ \\
\hline I have severe problems doing my usual activities & $175(7.2)$ & $(6.20$ to 8.27$)$ \\
\hline I am unable to do my usual activities & $39(1.6)$ & $(1.11$ to 2.11$)$ \\
\hline Missing & $2(0.1)$ & $(0.00$ to 0.20$)$ \\
\hline \multicolumn{3}{|l|}{ EQ-5D-5L pain/discomfort* } \\
\hline I have no pain or discomfort & $541(22.4)$ & (20.70 to 24.03$)$ \\
\hline I have slight pain or discomfort & $635(26.3)$ & $(24.50$ to 28.00$)$ \\
\hline I have moderate pain or discomfort & $670(27.7)$ & $(25.91$ to 29.48$)$ \\
\hline I have severe pain or discomfort & $470(19.4)$ & $(17.85$ to 21.01$)$ \\
\hline I have extreme pain or discomfort & $99(4.1)$ & $(3.30$ to 4.88$)$ \\
\hline Missing & $4(0.2)$ & $(0.00$ to 0.33$)$ \\
\hline \multicolumn{3}{|l|}{ EQ-5D-5L anxiety/depression* } \\
\hline I am not anxious or depressed & $1063(43.9)$ & $(41.97$ to 45.92$)$ \\
\hline I am slightly anxious or depressed & $722(29.8)$ & $(28.02$ to 31.67$)$ \\
\hline
\end{tabular}


Table 2 continued

\begin{tabular}{|c|c|c|}
\hline Assessment tool & $n$ or $n(\%)$ & Mean $(95 \% \mathrm{CI})$ score \\
\hline I am moderately anxious or depressed & $437(18.1)$ & (16.53 to 19.60$)$ \\
\hline I am severely anxious or depressed & $150(6.2)$ & $(5.24$ to 7.16$)$ \\
\hline I am extremely anxious or depressed & $44(1.8)$ & $(1.29$ to 2.35$)$ \\
\hline Missing & $3(0.1)$ & $(0.00$ to 0.26$)$ \\
\hline HIT-6 total score & 2415 & $65.2(65.0$ to 65.5$)$ \\
\hline \multicolumn{3}{|l|}{ HIT-6 total score grade* } \\
\hline Little or no impact & $29(1.2)$ & $(0.77$ to 1.63$)$ \\
\hline Some impact & $100(4.1)$ & (3.34 to 4.93$)$ \\
\hline Substantial impact & $157(6.5)$ & (5.51 to 7.47$)$ \\
\hline Severe impact & $2129(88.0)$ & (86.72 to 89.31$)$ \\
\hline Missing & $4(0.2)$ & $(0.00$ to 0.33$)$ \\
\hline mMIDAS total score & 2380 & $25.1(24.4$ to 25.9$)$ \\
\hline \multicolumn{3}{|l|}{ Modified MIDAS disability grade* } \\
\hline I & $160(6.6)$ & $(5.62$ to 7.60$)$ \\
\hline II & $76(3.1)$ & ( 2.45 to 3.84$)$ \\
\hline III & $162(6.7)$ & (5.70 to 7.69$)$ \\
\hline IV & $1982(81.9)$ & $(80.40$ to 83.47$)$ \\
\hline Missing & $39(1.6)$ & (1.11 to 2.11$)$ \\
\hline MSQ-RFR score & 2417 & $43.8(43.0$ to 44.6$)$ \\
\hline MSQ-RFP score & 2415 & $59.2(58.2$ to 60.1$)$ \\
\hline MSQ-EF score & 2416 & $50.5(49.4$ to 51.6$)$ \\
\hline WPAI percentage work time missed & 1587 & $15.6(14.3$ to 16.9$)$ \\
\hline WPAI percentage impairment while working & 1512 & $48.6(47.2$ to 49.9$)$ \\
\hline WPAI percentage overall work impairment & 1496 & $52.6(51.2$ to 54.0$)$ \\
\hline WPAI percentage activity impairment & 2410 & $56.4(55.3$ to 57.4$)$ \\
\hline HADS overall score & 2395 & $14.6(14.3$ to 15.0$)$ \\
\hline HADS anxiety & 2401 & $8.0(7.8$ to 8.2$)$ \\
\hline HADS depression & 2401 & $6.7(6.5$ to 6.8$)$ \\
\hline \multicolumn{3}{|l|}{ HADS anxiety subscale—categorical ${ }^{*}$} \\
\hline Below 11 & $1700(70.3)$ & $(68.46$ to 72.10$)$ \\
\hline 11 or above & $701(29.0)$ & (27.17 to 30.79$)$ \\
\hline Missing & $18(0.7)$ & (0.40 to 1.09$)$ \\
\hline
\end{tabular}


Table 2 continued

\begin{tabular}{lll}
\hline Assessment tool & $\boldsymbol{n}$ or $\boldsymbol{n}$ (\%) & Mean (95\% CI) score \\
\hline HADS depression subscale-categorical & & \\
Below 11 & $1923(79.5)$ & $(77.89$ to 81.10$)$ \\
11 or above & $478(19.8)$ & $(18.17$ to 21.35$)$ \\
Missing & $18(0.7)$ & (0.40 to 1.09$)$ \\
\hline
\end{tabular}

${ }^{*}$ Only $95 \%$ CI is reported. CIs were calculated using Wald formula/method

$C I$ confidence interval, $E F$ emotional function, EQ-5D-5L EuroQoL 5 dimensions, 5 levels, HADS Hospital Anxiety and Depression, HIT-6 headache impact test, $m M I D A S$ modified Migraine Disability Assessment, $M S Q$ migraine-specific quality of life, $N$ number of patients included to analyze the respective questionnaire, $P R O$ patient-reported outcome, $R F P$ role function-preventive, $R F R$ role function-restrictive, $V A S$ visual analog scale, WPAI work productivity and activity impairment

category, the burden was noticeably associated with PPTF (Table S2 in the supplementary material).

The disease-specific MSQ questionnaire indicated substantial effects of migraine on daily activities (MSQ Role Function Restrictive [MSQ-RFR]), social/work-related activities (MSQ Role Function Preventive [MSQ-RFP]), and emotional function (MSQ-EF). On a scale of $0-100$, with 100 reflecting the best imaginable health state, the mean (95\% CI) MSQ-RFR score was 43.8 (43.0-44.6), indicating a high interference of migraine with social and work-related activities. The mean MSQ-RFP score of 59.2 (58.2-60.1) indicated that patients had to cancel or needed help in their social and work-related lives because of migraine symptoms. The mean score in the MSQ-EF dimension was 50.5 (49.4-51.6), demonstrating the frustration or fear of patients of letting others down because of their migraines (Table 2). In the subgroup analyses, the MSQ scores across all three domains (RFR, RFP, and EF) tended to worsen with increasing MMD but not with PPTF (Table S2 in the supplementary material).

WPAI scores are expressed as percentages, with higher scores reflecting greater impairment and loss of productivity. For the overall part 2 population, the mean (95\% CI) WPAI-headache scores indicated a high level of impairment reflected in the percentage of work time missed (15.6\% [14.3-16.9]), percentage impairment while working $(48.6 \% \quad[47.2-49.9])$, and percentage overall work impairment $(52.6 \%$ [51.2-54.0]). In the subgroup analyses, the mean scores increased with increasing MMD within all domains, and an apparent additional effect of PPTF in the EM categories was observed (Table S2 in the supplementary material).

\section{Migraine Management}

A total of 1970 patients consulted centers with a dedicated headache clinic, whereas 432 consulted centers without one (but with at least one practitioner dedicated to headache management). The proportion of follow-up patients consulting centers with (1506/1970, 76.4\%) and without $(343 / 432,79.4 \%)$ a dedicated headache clinic was similar. However, a greater proportion of patients with difficult-to-treat migraine (CM or $\geq 2$ PPTFs) visited centers with a dedicated headache clinic compared with centers without one (Table S3 in the supplementary material).

\section{Healthcare Resource Utilization}

During the 3 months before study enrollment, the majority of patients $(58.0 \%$ [1403/2419]) had consulted a neurologist (mean visits [SD], 1.4 [1.2]) for their headache, $32.0 \%(773 / 2419)$ had consulted a primary care physician (mean [SD], 2.6 [3.1] times), and 27.5\% (666/2419) had consulted any alternative office-based 


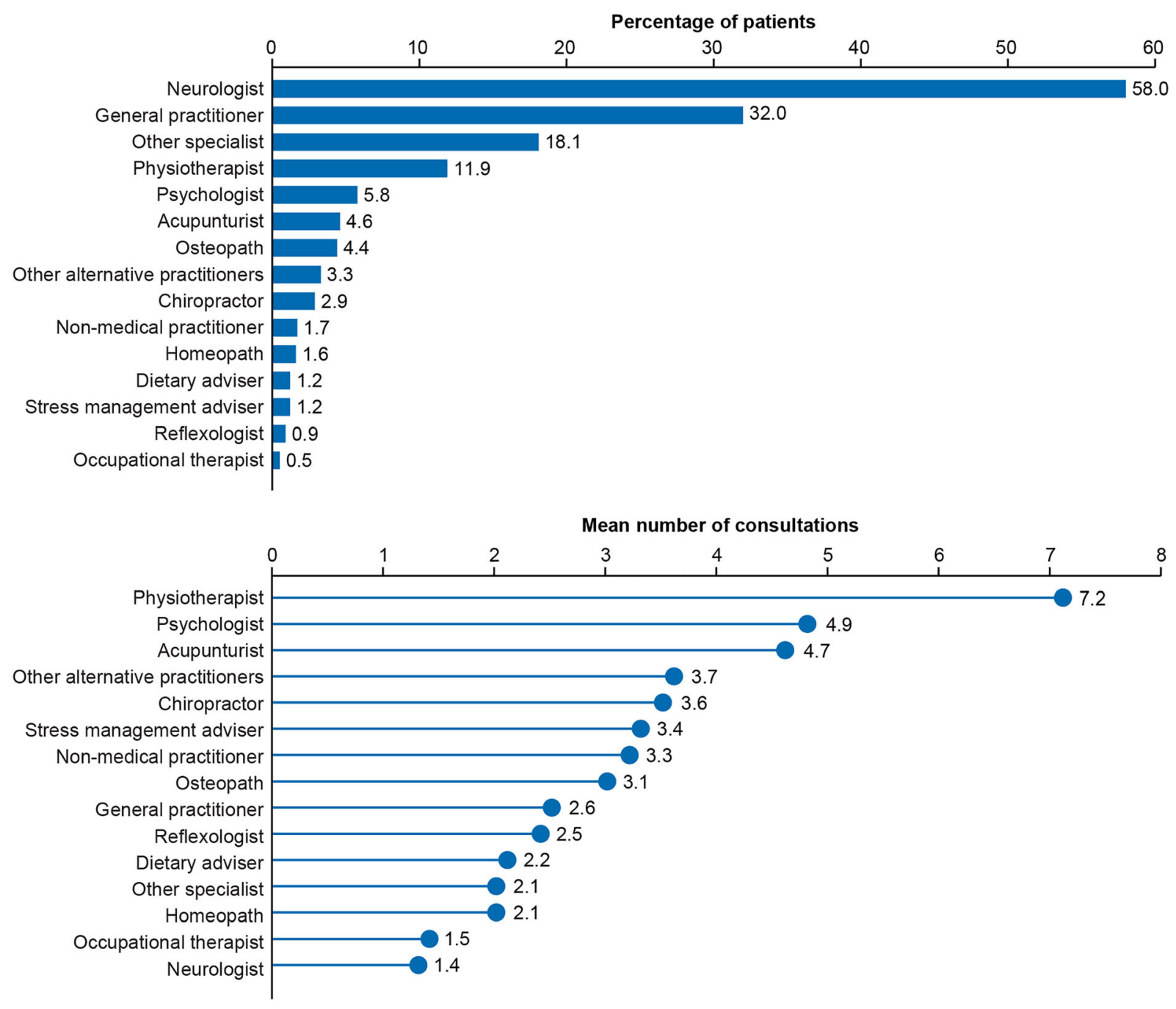

Fig. 4 Patients' consultations with specialists (a) and mean consultations $(\mathbf{b})$ in the previous 3 months. Percentages do not add up to $100 \%$ as patients may have consulted more than one specialist

practitioners (Fig. 4). Among the alternative office-based practitioners, physiotherapists were the most frequently consulted $(11.9 \%[288 /$ 2419]; mean visits [SD], 7.2 [6.7]). A small proportion of inpatients were treated for headaches by other hospital-based practitioners (Table S4 in the supplementary material). In the previous 12 months, $21.2 \%(514 / 2419)$ of patients reported visiting an ED for headache/migraine (mean visits [SD], 3.0 [5.5]) (Table 3), with marked differences in the proportion of patients visiting EDs across the participating countries (Table S5 in the supplementary material). Overall, 20.4\% (493/2419) and 11.6\% (281/ 2419) of patients underwent an MRI and CT scan, respectively, to investigate their headache/migraine during the previous 12 months. A total of $18.8 \%(455 / 2419)$ of patients were admitted as inpatients in the previous 12 months, $8.4 \%(203 / 2419)$ of whom were admitted for headache/migraine (Table 3).

Subgroup analyses showed that in the past 3 months, the proportion of patients who visited a neurologist increased with more frequent MMD; moreover, within each MMD category, patients with a higher number of PPTFs tended to visit the neurologist more often (Fig. 3Sa in the supplementary material). In the past 12 months, the proportion of patients who were hospitalized for their migraine was high among 
Table 3 Patients utilizing healthcare resources for their headache/migraine in the previous year

\begin{tabular}{lll}
\hline Healthcare resource & Part $\mathbf{2}(\boldsymbol{N}=\mathbf{2 4 1 9}), \boldsymbol{n}(\%)$ & Visits or investigations, mean $(\mathbf{S D})$ \\
\hline Emergency department & $514(21.2)$ & $3.0(5.5)$ \\
CT scan & $281(11.6)$ & $1.1(0.5)$ \\
MRI scan & $493(20.4)$ & $1.1(0.4)$ \\
Hospital admission & $203(8.4)$ & $1.5(2.3)$ \\
Hospital admission for any other reason & $252(10.4)$ & $1.4(1.3)$ \\
\hline
\end{tabular}

$C T$ computed tomography, $M R I$ magnetic resonance imaging, $N$ total number of patients in part $2, n$ number of patients with visits/investigations, $S D$ standard deviation

the CM subgroups, particularly in those with a high number of PPTFs (Fig. 3Sb). The proportion of patients who visited the ED for their headache/migraine in the previous 12 months increased with increases in MMD and further increased with the number of PPTFs within the EM subgroups (Fig. 3Sc).

\section{Comorbidities}

Overall, comorbidities were reported in $61.1 \%$ (1479/2419) of patients in part 2 and the mean (SD) number of comorbidities per patient was 1.3 (1.6). The most common comorbidities reported were psychiatric disorders (22.0\% [532/ 2419]), musculoskeletal and connective tissue disorders (13.3\% [322/2419]), gastrointestinal disorders (10.3\% [250/2419]), vascular disorders (10.3\% [249/2419]), and endocrine disorders (10.2\% [246/2419]). When analyzed according to subgroups, and in general, both the overall proportion of patients with comorbidities and the number of comorbidities per patient increased with increasing MMD and an increasing number of PPTFs within an MMD category (Table S6 in the supplementary material). Of note, the frequency of psychiatric comorbidities was consistently higher in patients with $\geq 4$ PPTFs.

\section{DISCUSSION}

This study identified a high unmet need among the BECOME population. In part 1 of the study, comprising patients visiting headache centers during a 3-month period, $62.2 \%$ of patients reported failure of at least one prior preventive therapy and $74.3 \%$ of patients reported $\geq 4 \mathrm{MMD}$, representing a high burden of the disease. Results from the PRO measures administered in part 2 of the study demonstrated that patients who had previously failed preventive treatments suffered severe impact of the migraine on their QoL, and on their personal, professional, and social activities. HRU assessment data also suggested a high burden of migraine management in terms of visits to physicians, use of $\mathrm{ED}$ and neuroimaging resources (MRI and CT scan), and hospital admissions.

Only a quarter $(24.4 \%$ [38/156]) of the participating centers in the BECOME study were equipped with multidisciplinary teams (Table S7 in the supplementary material), the gold standard for headache treatment, signifying an area of improvement for the specialist centers in Europe. Furthermore, the waiting period for a new patient to receive an appointment at these centers (mean of 3.3 months and maximum reported time of 18 months) reflects the challenges in gaining access to specialist care sites. These findings underline the need for appropriate referrals, a concern that has also been raised in other studies $[8,9]$.

In the BECOME study, three-quarters of the patients visiting headache centers for 3 months were eligible for preventive medication $(74.3 \%$ had $\geq 4 \mathrm{MMD})$. The high proportion of patients with documented PPTF $(62.2 \%$ of patients with $\geq 1$ PPTF in part 1 and $15.3 \%$ with $\geq 4$ PPTFs in part 2) and a quarter (23.6\%) of the part 2 
population with medication overuse headache demonstrates the difficulty in managing migraine and the need for treatment re-evaluation in patients with refractory disease. In addition, $82.6 \%$ of patients had visited any specialist at least once, $54.7 \%$ consulted at least two different specialists (many with two or more visits), $31.3 \%$ consulted three or more specialists, and $17.2 \%$ consulted more than four different specialists for their migraine, reflecting the high disease burden and the need for active management of this patient group.

In this study, six PRO measures were used to assess the burden of migraine on patients' lives. Three of these measures were generic and three were specific to migraine. The EQ-5D-5L questionnaire was used to assess the effect of migraine on general health status. Responses to this measure demonstrated that both MMD load and PPTF were associated with having at least moderate problems in performing usual activities and having at least moderate pain or discomfort. However, the MMD load, but not PPTF, was associated with being at least moderately anxious/depressed.

Responses to the MSQ questionnaire further indicated the substantial limitations and restrictions on daily activities and emotional well-being associated with migraine. Patients reported a severe impact of headache on daily life (average HIT-6 score 65.2 , with $88.0 \%$ of patients scoring in the most severe category) and severe migraine-related disability (average mMIDAS score 25.1), with the majority of the population $(81.9 \%)$ reaching a score for the latter that represented grade IV severity (score of 21 or higher). The severity of migraine, consistent across the mMIDAS and HIT- 6 measures, indicated that despite being managed at specialist headache centers, many patients were unable to find adequate and acceptable treatment options.

Results from the WPAI-headache questionnaire suggested a significant detrimental effect of migraine on the productivity of the working population in the participant countries, either due to absenteeism from work (mean score 15.6) or reduced productivity for those going to work (mean score 48.6). The economic burden due to lost productivity of the migraine population in their most productive age group is severe, as reported previously [10].

The association of both anxiety and depression with migraine has been previously reported $[11,12]$. In this study, the mean HADS depression was within the normal range for the overall study population (6.7), but the proportion of patients with a score of 11 or above (indicating depression) was $28.7 \%$ (289/1007) among patients with $\mathrm{CM}$ and $14 \%(85 / 605)$ among patients with HFEM. Additionally, the mean HADS anxiety subscale score (8.0) indicated the presence of anxiety in the overall population, with $35.8 \%(361 / 1007)$ of patients with CM and $27.3 \%(165 / 605)$ of patients with HFEM reporting a score of 11 or above. These findings were corroborated by the findings using the EQ-5D$5 \mathrm{~L}$ scale, where $26.1 \%$ of patients reported being at least moderately anxious or depressed.

Observations from the subgroup analyses suggested that a borderline abnormal score or a score of $>8$ on the HADS anxiety and HADS depression subscales was more strongly associated with headache frequency than with PPTF. The burden of disease, as measured using the HIT- 6 and mMIDAS scores, increased with increasing MMD, and within a given MMD category, the burden was noticeably associated with PPTFs. On the other hand, MSQ scores increased with increasing MMD, but little modulation by number of PPTFs was observed. The WPAI scores across all domains were found to be associated with MMD with no consistent additional effect of PPTFs.

Twenty-two percent of participants in the BECOME study had a psychiatric comorbidity and of this depression and anxiety accounted for $17 \%$. These data support the intrinsic link between migraine and psychiatric comorbidity. Subgroup analyses revealed that the frequency of psychiatric comorbidities was associated with both the number of MMD and the number of PPTFs. Concomitant treatment with antidepressants and anxiolytics was reported for $15.1 \% \quad(366 / 2419)$ and $4.9 \%(119 / 2419)$ of patients, respectively.

Part 2 of this study also included HRU assessment. A high variation in the frequency of ED visits, CT scans, and MRI scans was observed among the participating countries of the 
BECOME study (Table S5 in the supplementary material), highlighting the differences in healthcare systems and clinical practice across European countries, in line with data from previous studies [13-15]. Overall, in this study, we found high proportions of patients consulting for a follow-up visit (77.0\%) and reporting ED visits $(21.2 \%$; mean [SD] visits per patient, 3.0 [5.4]) for their migraine and hospitalization due to migraine (8.4\%; mean [SD], 1.5 [2.3]) in the previous 12 months. Among the subgroups analyzed, hospitalizations were particularly high among patients with CM, where $17.4 \%$ of patients with 4 PPTFs had been hospitalized an average of 1.5 times in the previous 12 months because of their migraine. Interestingly, the proportion of patients who visited ED was high in patients with EM, high MMD frequency, and high PPTF (HFEM/4 PPTF, 30.3\%). These findings are in line with the results from the My Migraine Voice survey, and add to the evidence on burden of disease among patients with migraine, and importantly, provide additional information on the burden associated with prior preventive treatment failures [16]. Subgroup analyses showed that, in general, HRU increased with an increasing number of PPTFs within any category of MMD.

The specific aim of this study was to assess the burden of migraine in patients with at least one PPTF visiting the specialist centers in Europe and Israel. Since the data were collected from headache specialist centers, the diagnosis of migraine is expected to be more accurate in this study than if carried out in a broader settings. This was a conscious decision to best ensure accurate assessment of true treatment failures as well as a correct attribution of burden and HRU to migraine, excluding confounding results from other headache disorders. However, this approach also introduces a limitation in that the data may not be representative of patients visiting general clinics. As with all noninterventional studies with self-reported elements, recall bias may be a factor in the data reported on PPTF, MMD, and PROs.

\section{CONCLUSIONS}

The BECOME study demonstrates the high prevalence of PPTF among patients with migraine who visited headache clinics and confirms the significant and increasing HRQoL and societal burden in patients with increasing disease severity due to migraine. Both PRO and HRU data revealed that the disease burden increases with MMD. Furthermore, our results indicate that the number of PPTFs add to the burden of migraine. Collectively, these findings demonstrate an unmet need for the management of difficult-to-treat migraine.

\section{ACKNOWLEDGEMENTS}

The authors thank the center participants, Ronald Zielman for his support on visualization of burden of migraine across the migraine spectrum and others involved in the study.

Funding. This study was funded by Novartis Pharma AG, Basel, Switzerland. The study sponsor participated in the study design, data collection, data review, data analysis and writing of the report. The Rapid Service Fee was funded by Novartis Pharma AG, Basel, Switzerland.

Medical Writing Assistance. Medical writing support for the manuscript was provided by Sashi Kiran Goteti, and Rohita Sri Gattoju of Novartis Healthcare Pvt. Ltd, Hyderabad, India, which was funded by Novartis Pharma AG, Basel, Switzerland, in accordance with Good Publication Practice (GPP3) guidelines (http:// www.ismpp.org/gpp3).

Authorship. All named authors meet the International Committee of Medical Journal Editors (ICMJE) criteria for authorship for this article, take responsibility for the integrity of the work as a whole, and have given their approval for this version to be published.

Authors' Contributions. Josefin Snellman and Shannon Ritter designed the study with 
input from Patricia Pozo-Rosich, David PB Watson, Charly Gaul, Christian Lucas, and Paolo Martelletti. Josefin Snellman and Shannon Ritter designed the survey and had primary responsibility for data collection. Shannon Ritter led the analysis with assistance from Josefin Snellman and Emma Ramsden. Patricia PozoRosich was the primary author; all other authors contributed to the writing of the manuscript. Patricia Pozo-Rosich is the guarantor and attests that all listed authors meet authorship criteria and that no others meeting the criteria have been omitted.

Prior Presentation. The majority of the data in the manuscript has not been presented previously. Aspects were presented at scientific congresses during 2018 at the European Headache Federation (EHF; Florence, Italy), during 2019 at the European Academy of Neurology (EAN; Oslo, Norway), American Headache Society (AHS; Philadelphia, Pennsylvania), The International Headache Congress (IHS, Dublin, Ireland), International Association for the Study of Pain (IASP; Amsterdam, Netherlands), Italian Society of Neurology (SIN; Bologna, Italy) and during 2020 at virtual meetings of the EAN, AHS, EHF, Association of British Neurologists $(\mathrm{ABN})$ and Migraine Trust International Symposium (MTIS).

Disclosures. All authors have completed the ICMJE uniform disclosure form at www.icmje. org/coi_disclosure.pdf. Patricia Pozo-Rosichreceived honoraria as a consultant and speaker during the last 5 years from Allergan, Almirall, Biohaven, Chiesi, Corpus, Eli Lilly, Medscape, Neurodiem, Novartis and Teva. Her research group has received research grants from Allergan and has received funding for clinical trials from Alder, electroCore, Eli Lilly, Teva, and Amgen/Novartis. She is a member of the Board of Trustees of the International Headache Society and a member of the Council of the European Headache Federation. She is on the editorial board of Revista de Neurologia. She is an associate editor for Cephalalgia, Frontiers of Neurology and The Journal of Headache and Pain. She is a member of the Clinical Trials Guidelines Committee of the International Headache
Society. She has edited the Guidelines for the Diagnosis and Treatment of Headache of the Spanish Neurological Society. She does not own stocks from any pharmaceutical company. Christian Lucas-collaborated as an expert, investigator or coordinator of clinical trials with Novartis, Teva, Sanofi, Grünenthal, Eli Lilly, Biogen, Lundbeck and Ethypharm. David PB Watson-received honoraria from Novartis, Teva, and Allergan in the last 12 months for consultancy and educational work. Charly Gaul (affiliation at the time of study as provided in the author list and now Professor at Headache Center Frankfurt, Germany)_received honoraria for consulting and lectures within the past 3 years from Allergan Pharma, Eli Lilly, Novartis Pharma, Hormosan Pharma, Grünenthal, Sanofi-Aventis, Weber \& Weber, Lundbeck and TEVA. He does not hold any stocks of pharmaceutical companies or medical device companies. Emma Ramsden-provides services to Novartis Pharma AG. Paolo Martelletti-received advisory board fees form Allergan, Eli Lilly, Novartis and Teva. Section editor, Medicine, Springer Nature Comprehensive Clinical Medicine; editor-in-chief, The Journal of Headache and Pain; Headache Books Series editor, Springer; and EU expert, European Medicine Agency. Former president of the European Federation and chairman of the School of Advanced Studies of the European Headache Federation. He does not hold any stocks of any pharmaceutical companies or medical device companies. Shannon Ritter and Josefin Snellman-employees and own stocks in Novartis.

Compliance with Ethics Guidelines. The study was approved by an independent ethics committee or relevant institutional review board at all participating centers (Table S9) and conducted in accordance with the ethical principles laid down in the Declaration of Helsinki. All participants provided informed consent before study initiation.

Data Availability. De-identified study data may be made available on request to researchers who submit a proposal that is approved by the principal investigator. 
Open Access. This article is licensed under a Creative Commons Attribution-NonCommercial 4.0 International License, which permits any non-commercial use, sharing, adaptation, distribution and reproduction in any medium or format, as long as you give appropriate credit to the original author(s) and the source, provide a link to the Creative Commons licence, and indicate if changes were made. The images or other third party material in this article are included in the article's Creative Commons licence, unless indicated otherwise in a credit line to the material. If material is not included in the article's Creative Commons licence and your intended use is not permitted by statutory regulation or exceeds the permitted use, you will need to obtain permission directly from the copyright holder. To view a copy of this licence, visit http://creativecommons.org/licenses/by$\mathrm{nc} / 4.0 /$.

\section{REFERENCES}

1. GBD 2016 Disease and Injury Incidence and Prevalence Collaborators. Global, regional, and national incidence, prevalence, and years lived with disability for 328 diseases and injuries for 195 countries, 1990-2016: a systematic analysis for the Global Burden of Disease Study 2016. Lancet. 2017;390(10100):1211-59.

2. Steiner TJ, Stovner LJ, Vos T, Jensen R, Katsarava Z. Migraine is first cause of disability in under 50s: will health politicians now take notice? J Headache Pain. 2018;19(1):17.

3. Lipton RB, Bigal ME, Diamond M, et al. Migraine prevalence, disease burden, and the need for preventive therapy. Neurology. 2007;68(5):343-9.

4. Adams AM, Serrano D, Buse DC, et al. The impact of chronic migraine: the Chronic Migraine Epidemiology and Outcomes (CaMEO) Study methods and baseline results. Cephalalgia. 2015;35(7):563-78.

5. Blumenfeld AM, Varon SF, Wilcox TK, et al. Disability, HRQoL and resource use among chronic and episodic migraineurs: results from the International Burden of Migraine Study (IBMS). Cephalalgia. 2011;31(3):301-15.
6. Steiner TJ, Stovner LJ, Katsarava Z, et al. The impact of headache in Europe: principal results of the Eurolight project. J Headache Pain. 2014;15:31.

7. Headache Classification Committee of the International Headache Society. The International Classification of Headache Disorders, 3rd edition (beta version). Cephalalgia. 2013;33(9):629-808.

8. Steiner TJ, Jensen R, Katsarava Z, et al. Aids to management of headache disorders in primary care (2nd edition): on behalf of the European Headache Federation and Lifting The Burden: the Global Campaign against Headache. J Headache Pain. 2019;20(1):57.

9. Kainth P, Niamh M, SIlvia R, et al. Management of migraine and the accessibility of specialist carefindings from a multi-national assessment of 28 healthcare networks. Eur Neurol Rev. 2018;13(2): 103-10.

10. Lanteri-Minet M, Duru G, Mudge M, Cottrell S. Quality of life impairment, disability and economic burden associated with chronic daily headache, focusing on chronic migraine with or without medication overuse: a systematic review. Cephalalgia. 2011;31(7):837-50.

11. Lanteri-Minet M, Radat F, Chautard MH, Lucas C. Anxiety and depression associated with migraine: influence on migraine subjects' disability and quality of life, and acute migraine management. Pain. 2005;118(3):319-26.

12. Amoozegar F, Patten SB, Becker WJ, et al. The prevalence of depression and the accuracy of depression screening tools in migraine patients. Gen Hosp Psychiatry. 2017;48:25-31.

13. Katsarava Z, Mania M, Lampl C, Herberhold J, Steiner TJ. Poor medical care for people with migraine in Europe-evidence from the Eurolight study. J Headache Pain. 2018;19(1):10.

14. Schramm S, Uluduz D, Gouveia RG, et al. Headache service quality: evaluation of quality indicators in 14 specialist-care centres. J Headache Pain. 2016;17(1):111.

15. Sanderson JC, Devine EB, Lipton RB, et al. Headache-related health resource utilisation in chronic and episodic migraine across six countries. J Neurol Neurosurg Psychiatry. 2013;84(12):1309-17.

16. Martelletti $P$, Schwedt TJ, Lanteri-Minet $M$, et al. My Migraine Voice survey: a global study of disease burden among individuals with migraine for whom preventive treatments have failed. J Headache Pain. 2018;19(1):115. 